\title{
AISLAMIENTO DE Bacillus SOLUBILIZADORES DE FOSFATOS ASOCIADOS AL CULTIVO DEL ARROZ ${ }^{1}$
}

\author{
Berto Tejera-Hernández ${ }^{2}$, Mayra Heydrich-Pérez $z^{2}$,Marcia M. Rojas-Badía ${ }^{2}$
}

\section{RESUMEN}

Aislamiento de Bacillus solubilizadores de fosfatos asociados al cultivo del arroz. El objetivo de este trabajo fue determinar la capacidad de bacterias del género Bacillus provenientes del cultivo del arroz (Oryza sativa L.) en solubilizar fosfatos. Los aislamientos se obtuvieron utilizando el modelo Microcosmos de los cultivares Iacuba 30 y Perla, en el año 2008 en Bauta, La Habana, Cuba. Hubo 58 aislados, tanto rizosféricos como endófitos de la raíz y de la parte aérea, que presentaban características distintivas del género Bacillus, como respuesta positiva a la tinción de Gram, forma bacilar y formación de endospora. Se realizó la detección cualitativa en cuanto a la capacidad de solubilización de fosfatos. Solo 19 aislamientos mostraron solubilización en medio sólido NBRIP y en algunos casos cambiaron la coloración del medio de azul a amarillo, lo que indica la producción de ácidos. Se seleccionaron los positivos para la cuantificación en medio líquido. Las cepas mostraron un comportamiento diferente cuando se midió el fosfato solubilizado a distintos tiempos, variando el máximo de solubilización.

Palabras claves: Bacilli, fosfatos inorgánicos, Oryza

\begin{abstract}
Phosphate solubilization by rice associate Bacillus. The objective pursued with this study was to isolate Bacillus bacteria from rice (Oryza sativa L.) and determine its potential for phosphate solubilization. Isolates were obtained using the Microcosm model from Iacuba 30 and Perla cultivar, established by Bauta (2008) in La Habana, Cuba. Fifty eight isolates were obtained from the rice rhizosphere and inside of the roots and leaves with characteristics typical of Bacillus, such as Gram positive, rod cells and endospore formation. The qualitative capacity of these isolates for phosphate solubilization was assayed in solid NBRIP medium; the results revealed that only 19 strains solubilized inorganic phosphate and some of them changed the medium color from blue to yellow, presumably due to acid excretion. Positives were selected for assay in liquid medium, and they showed different responses in terms of time and maximum phosphate solubilization.
\end{abstract}

Keywords: Bacilli, inorganic phosphate, Oryza sativa L. sativa $\mathrm{L}$. 


\section{INTRODUCCIÓN}

El fósforo es uno de los nutrientes más limitantes en el crecimiento de las plantas. Dentro de las funciones que le han sido atribuidas se encuentran la captación, almacenamiento y transferencia de energía además de formar parte de macromoléculas tales como ácidos nucleicos y fosfolípidos presentes en la membrana citoplasmática. Una gran porción de fosfatos inorgánicos aplicados al suelo como fertilizantes son inmovilizados después de su aplicación, permaneciendo de forma inasequible para la planta (Rengel y Marschner 2005). Esto permite predecir que aquellos microorganismos que colonicen la raíz de estas plantas y que tengan la capacidad de solubilizar el fósforo, actúen como promotores del crecimiento vegetal (Idriss et al. 2002).

Las formas inorgánicas de fósforo son solubilizadas por un grupo de microorganismos heterotróficos que excretan ácidos orgánicos, los cuales disuelven minerales ricos en fósforo o quelan cationes unidos al fósforo liberando a este a la solución (He et al. 2002). El género Bacillus es uno de los más estudiados respecto a esta capacidad y se plantea como mecanismo principal de solubilización, la excreción al medio de ácidos orgánicos (Rajankar et al. 2007).

El arroz constituye uno de los alimentos más importantes para la población mundial y su producción se encuentra concentrada fundamentalmente en Asia. Esto hace que si algunos de estos países productores vieran afectadas su producción, también se afectaría el consumo mundial (FAO 2007). El crecimiento de la población mundial es un factor que influye seriamente en el incremento de la demanda de arroz (United Nations Population Fund 2011), lo que ha conllevado a un incremento sustancial de la demanda y uso de fertilizantes químicos (Witt et al. 2009).

Es por esto que aumentar la cantidad de países productores y la producción constituye un reto para la humanidad en diversos sentidos. Una de las alternativas que se han encontrado para incrementar la producción y a la vez proteger el medio ambiente, es el empleo de bacterias solubilizadoras de fosfatos en cultivos de interés económico. Según plantean Salimpour et al. (2010), la aplicación de estos inoculantes puede potenciar la eficiencia del uso del fósforo natural o sintético y optimizar la aplicación de fertilizantes en este cultivo.
El objetivo de este trabajo fue determinar la capacidad de bacterias del género Bacillus provenientes del cultivo del arroz (Oryza sativa L.) en solubilizar fosfatos.

\section{MATERIALES Y MÉTODOS}

\section{Aislamiento}

Se realizaron tres muestreos del género Bacillus en el cultivo del arroz, los cultivares Iacuba 30 y Perla, entre los meses de febrero y marzo del año 2008 en campos del Instituto de Investigaciones del Arroz, en Bauta, La Habana (Cuba), que nunca habían sido biofertilizados. Las muestras se tomaron de plantas de aproximadamente quince días después de germinadas. La toma de estas se realizó según el modelo propuesto por Hernández (2002).

Para el aislamiento se utilizó el modelo microcosmos (Kabir et al. 1995). Se desinfectaron las semillas de arroz (Oryza sativa L.) siguiendo la metodología propuesta por García et al. (2008). Las semillas desinfectadas se colocaron en cámaras húmedas entre tres y cinco días para su pregerminación y se sembraron en la columna con suelo rizosférico de este cultivo, además se le añadió agua destilada estéril con el objetivo de mantener la humedad necesaria para la germinación de la semilla. Las columnas se colocaron a temperatura ambiente durante catorce días, en un fotoperíodo de ocho horas de luz y catorce de oscuridad.

En condiciones asépticas se tomaron las plántulas para separar la raíz de la parte aérea para el aislamiento de los endófitos de estas zonas de la planta. Para aislar rizobacterias del género Bacillus se tomó un gramo del suelo rizosférico y se adicionó a un tubo que contenía $9 \mathrm{ml}$ de solución salina $(\mathrm{NaCl}$ al $0,85 \%)$, se agitó vigorosamente con un agitador (Genie). Seguidamente se hicieron diluciones decimales seriadas hasta $10^{-6}$ en solución salina fisiológica estéril al $0,85 \%$ que se calentaron a $80^{\circ} \mathrm{C}$ durante 30 minutos y se sembraron por diseminación en medio Agar Triptona Soya (TSA, OXOID) y se incubaron a $37^{\circ} \mathrm{C}$ durante 24 horas.

Para el aislamiento de microorganismos endófitos se realizó la desinfección (García 2008) y la posterior maceración de los tejidos aéreos y radiculares en solución salina. Se tomó $1 \mathrm{ml}$ de macerado y se adicionaron a un tubo con $9 \mathrm{ml}$ de solución salina estéril, 
se agitó vigorosamente y a partir de este se realizaron diluciones decimales seriadas. Posteriormente, se procedió de la forma descrita para obtener representantes del género Bacillus.

A las 24 horas de incubación, a partir de las placas de TSA con crecimiento, se tomaron muestras de las colonias con características diferentes, teniendo en cuenta forma, tamaño, color, bordes, elevación, y se pasaron a placas por agotamiento para su purificación. Se realizó la observación microscópica con tinción de Gram para comprobar la pureza y determinar las características micromorfológicas y tintoriales de los mismos, así como la presencia de esporas. Las cepas aisladas se conservaron en tubos con agar nutriente y en glicerol al $20 \%$ a $-20^{\circ} \mathrm{C}$.

\section{Determinación de la solubilización de fosfatos}

La determinación cualitativa de la solubilización de fosfatos se llevó a cabo por siembra directa en el medio de cultivo NBRIP sólido (Glucosa $10 \mathrm{~g}, \mathrm{Ca}_{3} \mathrm{PO}_{4}$ $5 \mathrm{~g}, \mathrm{MgCl}_{2} 6 \mathrm{H}_{2} \mathrm{O} 5 \mathrm{~g} 0,25 \mathrm{~g}, \mathrm{KCl} 0,2 \mathrm{~g},\left(\mathrm{NH}_{4}\right)_{2} \mathrm{SO}_{4} 0.1 \mathrm{~g}$, Agar 20g, $\mathrm{H}_{2} \mathrm{O}$ destilada 1L) (Nautiyal 1999) al cual se le añadió azul de bromofenol como indicador de $\mathrm{pH}$ a una concentración de $0,025 \mathrm{~g} / \mathrm{l}$, lo cual permite observar la producción de ácido por un cambio de coloración de azul a amarillo. Las placas se incubaron a $37^{\circ} \mathrm{C}$ durante 24 horas.

\section{Cuantificación de la solubilización de fosfatos}

Para la cuantificación de fosfato soluble en medio NBRIP líquido se siguió la metodología utilizada por Alikhani et al. (2007) y la misma se realizó a 0,24, 48 y 42 horas de incubación a $37^{\circ} \mathrm{C}$ y bajo condiciones de agitación y por triplicado. Se ajustó la concentración celular de $10^{8} \mathrm{cel} / \mathrm{ml}$ de los aislados en solución salina al $0,85 \%$ tomando como referencia el tubo 0,5 de la escala Mc Farland (NCCSL 1993).

\section{RESULTADOS Y DISCUSIÓN}

\section{Obtención y selección de los aislados}

Se obtuvieron 58 aislados, distribuidos entre la rizosfera y el interior de raíces y hojas, en los dos cultivares de arroz muestreados: IACuba 30 y Perla (Figura 1).

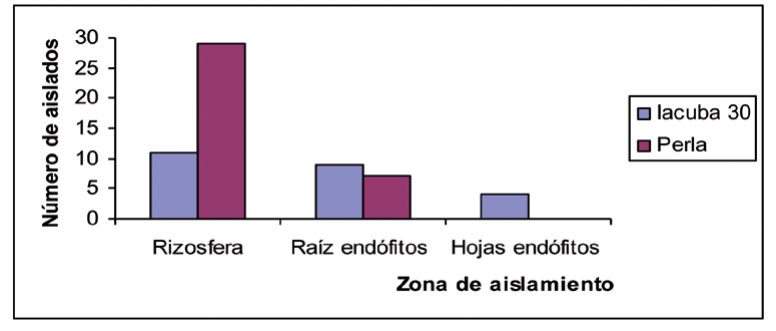

Figura 1. Distribución de los 58 aislamientos del género Bacillus en plantas de arroz de los cultivares IACuba 30 y Perla, obtenidos de las diferentes zonas. Bauta, La Habana, Cuba. 2008.

El modelo microcosmos, empleado para el aislamiento de microorganismos asociados a plantas, tiene como ventaja que simula las condiciones de campo, además los exudados radicales liberados por la planta permiten la quimiotaxis positiva de los microorganismos hacia la raíz y el aislamiento de microorganismos rizosféricos y autóctonos (Kabir et al. 1995).

La presencia de diferentes géneros bacterianos asociados a plantas de arroz cultivados en este mismo tipo de suelo y localidad, se ha informado previamente por Rives et al. (2006), entre los que se incluyen Azospirillum, Herbaspirillum, Azotobacter, Burkholderia y Pseudomonas, así como especies del género Bacillus (Beneduzi et al. 2008).

Se obtuvieron 34 aislamientos del cultivar Perla y 24 del cultivar IACuba 30, distribuidos entre 36 de la rizosfera, 16 del interior de la raíces y 4 del interior de las hojas. La mayoría de los aislamientos obtenidos son rizosféricos donde existe mayor diversidad de microorganismos que en el interior de la planta. Estos microorganismos endófitos establecen una relación más estrecha con su hospedero, lo que implica un reconocimiento a nivel molecular mucho más específico (Bai et al. 2003). En este sentido, también se plantea que estos microorganismos tienen ventajas competitivas respecto a aquellos que habitan en la rizosfera, debido a que existe una mayor disponibilidad de nutrientes dentro de la planta (James 2000); además estos microorganismos se encuentran menos expuestos a las condiciones adversas del medio ambiente (MuñozRojas y Caballero-Mellado 2003).

Para la selección de las cepas se tuvo en cuenta que las bacterias crecieran después del tratamiento a $80^{\circ} \mathrm{C}$ durante $30 \mathrm{~min}$, la respuesta a la tinción de Gram, la forma y la presencia de endosporas, características 
que se pueden observar en el Cuadro 1 y que dirigen la selección hacia el género Bacillus, lo que habría que corroborar posteriormente por otras metodologías.

La diversidad en cuanto a hábitat de este género se ha demostrado por la predominancia del mismo entre los bacilos Gram positivos aislados en suelos cultivados y dedicados al pasto sin prácticas de arado (Garbeva et al. 2008). En este sentido, se plantea que los géneros Bacillus y Pseudomonas predominan en la rizosfera por encima del resto de los géneros asociados a la misma (Adesemoye et al. 2008).

\section{Determinación cualitativa de la capacidad de solu- bilizar fosfato inorgánico}

Varios estudios han puesto en evidencia la capacidad solubilizadora de fosfatos por diferentes especies microbianas (Khan et al. 2010). Entre las bacterias destacan los géneros: Pseudomonas, Bacillus, Rhizobium, Burkholderia, Achromobacter, Agrobacterium, Microccocus, Aerobacter, Flavobacterium, Enterobacter, Pantoea, Klebsiella, Rhodobacter, Arthrobacter, Serratia y Erwinia (Patiño 2010).

En este experimento la solubilización de fosfato inorgánico se puso de manifiesto por la formación de un halo transparente en el medio NBRIP para los aislados RI2, RI7, RI8, RI11, EAI2, EAI4, EAI5, RP1, RP3, RP5, RP6, RP11, RP12, RP17, RP19, RP20, RP28, ERP2, ERP6, ERP7. Esto significa que el 29,31\% de los aislados tienen esta capacidad. En la Figura 2 se observa alrededor del crecimiento microbiano de los aislados RP19, RP20, RP12 y ERP2 una zona clara, que evidencia la solubilización de fosfatos. Este cambio de coloración ocurrió alrededor del crecimiento

Cuadro 1. Características micromorfológicas de los aislamientos del género Bacillus obtenidos a partir de los cultivares de arroz (Oryza sativa L.). Bauta, La Habana, Cuba. 2008.

\begin{tabular}{|c|c|c|c|c|}
\hline \multicolumn{4}{|c|}{ Aislados } & \multirow[t]{2}{*}{ Micromorfología } \\
\hline RI3 & RP1 & ERI1 & ERP2 & \\
\hline RI4 & RP9 & ERI2 & ERP5 & \multirow{5}{*}{$\begin{array}{c}\text { Bacilos finos y cortos, } \\
\text { Gram positivos, esporulados }\end{array}$} \\
\hline RI6 & RP14 & ERI3 & ERP6 & \\
\hline RI9 & RP15 & ERI5 & EAI2 & \\
\hline \multirow[t]{2}{*}{ RP29 } & RP16 & ERI10 & EAI3 & \\
\hline & RP17 & EAI5 & EAI4 & \\
\hline RI1 & RI11 & RP20 & ERP3 & \multirow{4}{*}{$\begin{array}{l}\text { Bacilos medianos y finos, } \\
\text { Gram positivos, esporulados }\end{array}$} \\
\hline RI5 & ERI8 & RP21 & ERP7 & \\
\hline RI8 & & RP22 & RP24 & \\
\hline RI10 & & RP23 & RP25 & \\
\hline \multirow[t]{2}{*}{$\mathrm{RI} 2$} & $\mathrm{RP} 2$ & RP19 & & \multirow{2}{*}{$\begin{array}{l}\text { Bacilos grandes y gruesos, } \\
\text { Gram positivos, esporulados }\end{array}$} \\
\hline & RP10 & RP27 & & \\
\hline RP11 & RP13 & $\mathrm{RP} 4$ & RP6 & \multirow{3}{*}{$\begin{array}{l}\text { Bacilos grandes y finos, } \\
\text { Gram positivos, esporulados }\end{array}$} \\
\hline \multirow[t]{2}{*}{ RP5 } & RP3 & $\mathrm{RP7}$ & RP12 & \\
\hline & & & ERP4 & \\
\hline RP18 & RP28 & ERI7 & ERP1 & $\begin{array}{l}\text { Bacilos medianos y gruesos, } \\
\text { Gram positivos, esporulados }\end{array}$ \\
\hline RI7 & ERI4 & ERI6 & & $\begin{array}{l}\text { Bacilos gruesos y cortos, } \\
\text { Gram positivos, esporulados }\end{array}$ \\
\hline
\end{tabular}




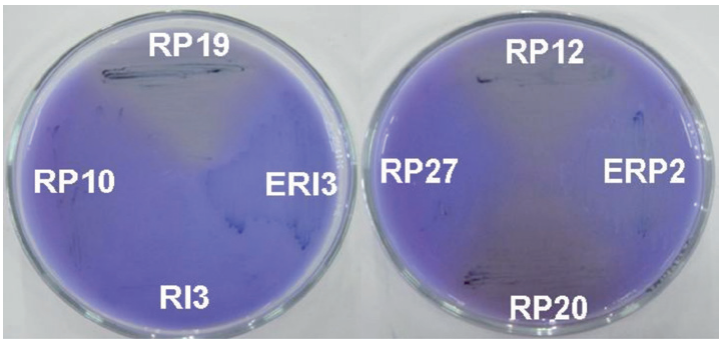

Figura 2. Halos de solubilización de fosfatos inorgánicos en medio NBRIP por aislamientos del género $\mathrm{Baci}$ llus obtenidos del cultivo del arroz. ERP (aislado endófito de rizosfera de Perla), RP (aislados de rizosfera de Perla). Bauta, La Habana, Cuba. 2008.

de las cepas RP19, RP12 y RP20, lo que se encuentra relacionado con el mecanismo a través del cual se lleva a cabo la solubilización de fosfatos.

Uno de los principales mecanismos de la solubilización de fosfatos es a través de la excreción de ácidos orgánicos al medio (Rodríguez y Fraga 1999). Esto ocurre debido a que se lleva a cabo un intercambio catiónico que permite que el fosfato insoluble se convierta a formas solubles, por lo que el mismo podría estar disponible para la planta. Dentro de los ácidos orgánicos excretados más comunes se encuentran el ácido cítrico, ácido láctico, ácido succínico y el ácido propiónico, siendo este último uno de los principales compuestos utilizados por especies como Bacillus megaterium para llevar a cabo la solubilización de fosfatos (Chen et al. 2006).

\section{Determinación cuantitativa de la capacidad de so- lubilizar fosfato inorgánico}

Las concentraciones de fosfato soluble variaron entre las 0,23 y $11,58 \mathrm{mg} / 1$. Los aislados RP20 y RP12 se destacan por su capacidad de solubilizar fosfato a las 48 horas de crecimiento (Figura 3).

En algunos aislados que resultaron ser positivos ante la determinación cualitativa como RI2, RP11, RP17y RP28 solo se encontró fosfato soluble a las 24 horas de incubación y en bajas concentraciones. Esto podría estar relacionado con el hecho de que estos microorganismos llevan a cabo el proceso de solubilización de fosfatos para potenciar el crecimiento al inicio de este o que el estado del medio de cultivo influya en la producción de los metabolitos encargados de este proceso.

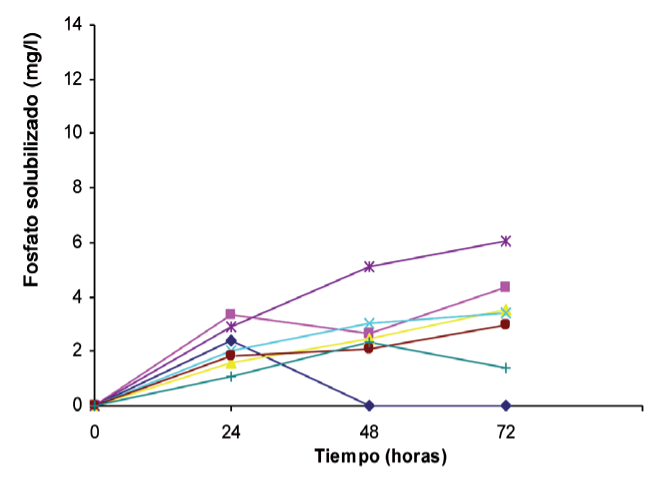

A

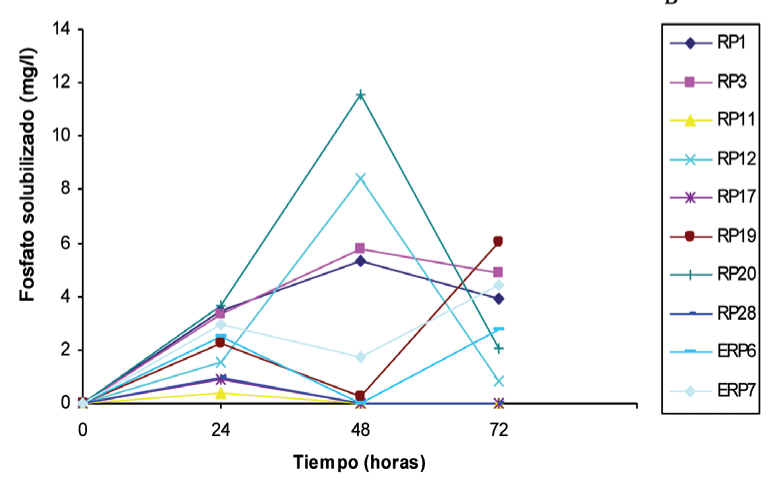

Figura 3. Dinámica de solubilización de fosfato en el medio NBRIP de los aislamientos de Bacillus, obtenidos en arroz, cultivares IACuba30 (A) y Perla (B). Bauta, La Habana, Cuba. 2008.

$\mathrm{Al}$ analizar la dinámica de solubilización de fosfatos, se puede observar que los aislamientos presentan un comportamiento diferente. EAI5, RP1, RP3, RP12 y RP20 tuvieron el máximo de solubilización a las 48 horas, mientras que RI11, RI7, RI8, EAI2, EAI4, RP19, ERP6, ERP7 a las 72 horas, el cual fue el tiempo máximo del experimento.

En una etapa inicial (hasta las 48 horas de incubación) se solubilizó el fosfato para consumirlo posteriormente. Algunos microorganismos exhiben represión de actividad solubilizadora ante la presencia de fósforo soluble y en otros no ocurría represión (Mikanova et al. 1997).

En algunas cepas de Bacillus, la mayor solubilización ocurrió después de las 18 a 24 horas del crecimiento bacteriano, correspondió con la fase logarítmica del crecimiento, mientras que en otras a partir de las 18 horas comenzó un decrecimiento, sin encontrar alguna explicación a ello (Vázquez et al. 2000). Este 
hecho también ocurre con las cepas estudiadas pero después de las 48 horas, lo que probablemente se deba al consumo de fósforo por el mismo microorganismo en el medio de cultivo o que el fósforo disponible regule el proceso de solubilización en algunas cepas $\mathrm{y}$ en otras no.

En especies del género Bacillus se ha demostrado la capacidad de los mismos para solubilizar fosfatos, esto ha sido relacionado con su actividad promotora del crecimiento vegetal en diferentes cultivos de interés económico como el maíz y el trigo (Egamberdiyeva et al. 2004). En este sentido se ha informado acerca de esta capacidad en especies como B. subtilis y B. megaterium (Rajankar et al. 2007). Esta última especie también ha sido aislada de la planta Cicer arietinum demostrando una alta eficiencia solubilizadora de fosfato en medio Pikovskaya's relacionado con efecto positivo sobre el crecimiento radicular (Sharma et al. 2007).

Por otra parte, se sabe que especies de este género como B. amyloliquefaciens aislado de rizosfera de papa solubilizan fosfato en el mismo medio de cultivo utilizado en este trabajo (Calvo et al. 2010). Además se informa que $B$. circulans aislado de la rizosfera de manzana solubiliza fosfatos en medio Pikovskaya combinado con la producción de ácido indolacético y sideróforos (Metha et al. 2010).

Algunas especies como $B$. liqueniformis y $B$. amyloliquefaciens solubilizan fosfatos a través de la excreción de una mezcla de ácidos orgánicos como el ácido láctico, isovalérico, isobutírico y acético (Metha y Nautiyal 2001).

Otros mecanismos involucran el intercambio catiónico, permitiendo que el fosfato insoluble se convierta a formas solubles por lo que el mismo podría estar disponible para la planta (Khan et al. 2009). Por otra parte, se ha demostrado que algunas especies como B. subtilis y B. megaterium se destacan por ser buenos solubilizadores de fósforo inorgánico (Rajankar et al. 2007).

Al analizar los porcentajes de cepas con esta capacidad se observa que es ligeramente mayor en cepas rizosféricas $(36,8 \%)$, debido a que estos microorganismos se encuentran en un ambiente donde la mayor cantidad de fosfato se presentan en formas insolubles (Goldstein 1994), por lo que tendrían que haber evolucionado hacia la utilización de esta fuente de nutrientes en las condiciones en que normalmente se encuentran en el suelo. En el caso del género $B a$ cillus se plantea que son microorganismos endófitos facultativos por lo que el hallazgo de microorganismos endófitos solubilizadores de fosfato, en este caso el $25 \%$, permite predecir que pudieron estar en contacto con fuentes de fosfato insoluble del suelo en algún momento de su ciclo de vida.

Se identificaron 19 cepas de Bacillus con la capacidad de solubilizar fosfatos inorgánicos lo que demuestra sus potencialidades en la estimulación del crecimiento vegetal. Estas pudieran contribuir positivamente al crecimiento de la planta como ocurre en el algodón (Qureshi et al. 2012), Kiwi (Erturk et al. 2010), tomate (Felici et al. 2008) y algunos vegetales (Ademoseye et al. 2008), entre otros cultivos.

\section{LITERATURA CITADA}

Adesemoye, AO; Obini, M; Ugoji, EO. 2008. Comparison of plant growth-promotion with Pseudomonas aeruginosa and Bacillus subtilis in three vegetables. Brazilian Journal of Microbiology 39:423-426.

Alikhani, HA; Rastin-Saleh, N; Antoun, H. 2007. Phosphate solubilization activity of rhizobia native Iranian soils. In Velázquez, E.; Rodríguez-Barrueco, C. eds. First International Meeting on Microbial Phosphate Solubilization. Springer, Netherlands. p. 35-41.

Bai, Y; Zhou, X; Smith, DL. 2003. Enhanced soybean plant growth resulting from coinoculation of Bacillus strains with Bradyrhizobium japonicum. Crop Sci. 43:1774-1781.

Beneduzi, A; Peres, D; Vargas, LK; Bodanese-Zanettini, MH; Passaglia, LMP. 2008. Evaluation of genetic diversity and plant growth promoting activities of nitrogen-fixing bacilli isolated from rice fields in South Brazil. Applied Soil Ecology 39:311-32.

Calvo, P; Ormeño-Orrillo, E; Martínez-Romero, E; Zúñiga, D. 2010. Characterization of Bacillus isolates of potato rhizosphere from andean soils of Perú and their potential PGPR characteristics. Brazilian Journal of Microbiology 41:899-906.

Chen, YP; Rekha, PD; Arun, AB; Shen, FT; Lai, W; Young, CC. 2006. Phosphate solubilizing bacteria from subtropical soil and their tricalcium phosphate solubilizing abilities. Applied Soil Ecology 34:33-41.

Egamberdiyeva, D; Juraeva, D; Poberejskaya, S; Myachina, O; Teryuhova, P; Seydalieva, L; Aliev, A. 2004. Improvement of wheat and cotton growth and nutrient uptake by phosphate solubilizing bacteria. In 26 th 
Southern Conservation Tillage Conference. Raleigh, North Carolina, USA. p. 58-66.

Erturk, Y; Ercisli. S; Haznedar, A; Cakmakci, R. 2010. Effects of plant growth promoting rhizobacteria (PGPR) on rooting and root growth of kiwifruit (Actinidia deliciosa) stem cuttings. Biol Res 43:91-98.

FAO (Food and Agriculture Organization of the United Nations). 2007. FAO production yearbook. In FAO rice market monitor, trade and markets division, Food and Agriculture Organization of the United Nations. Roma, Italia. Volume X Issue No. 3 (en línea). Consultado 13 mayo 2010. Disponible en http://www. fao.org

Felici, C; Vettori, L; Giraldi, E; Forino, LMC; Toffanin, A; Tagliasacchi, AM; Nuti, M. 2008. Single and co-inoculation of Bacillus subtilis and Azospirillum brasilense on Lycopersicon esculentum: Effects on plant growth and rhizosphere microbial community. Applied Soil Ecology 40:260-270.

Garbeva, P; Van Elsas, JD; Van Veen, JA. 2008. Rhizosphere microbial community and its response to plant species and soil history. Plant Soil 302:19-32.

García, JC; Hernández, A; Acebo, Y; Rives, N. 2008. Obtención de un nuevo método de desinfección de semillas de arroz. Cultivos Tropicales 29:55-59.

Goldstein, AH. 1994. Involvement of the quinoprotein glucose dehydrogenises in the solubilization of exogenous phosphates by gram-negative bacteria. In Torriani Gorini, A; Yagil, E; Silver, S. eds. Phosphate in Microorganisms: Cellular and Molecular Biology. Washington, D. C. USA, ASM Press. p. 197-203.

He, ZL; Bian, W; Zhu, J. 2002. Screening and identification of microorganisms capable of utilizing phosphate adsorbed by goethite. Comm. Soil Sci. Plant Anal. 33:647-663.

Hernández, A. 2002. Obtención de un biopreparado a partir de rizobacterias asociadas al cultivo del maíz (Zea mays L.). Tesis de doctorado. La Habana, Cuba, Facultad de Biología, Universidad de La Habana. 93 p.

Idriss, EE; Makarewicz, O; Farouk, A; Rosner, K; Greiner, R; Bochow, H; Richter, T; Borriss, R. 2002. Extracellular phytase activity of Bacillus amyloliquefaciens FZB45 contributes to its plant-growth-promoting effect. Microbiology 148:2097-2109.

James, EK; Gyaneshwar, P; Barraquio, WL; Mathan, N; Ladha, J. 2000. Endophytic diazotrophs associated with rice. In Ladha, JK; Reddy, PM. eds. The quest for nitrogen fixation in rice. International Rice Research Institute, Los Baños, Laguna, Philippines. p. 119-140.

Kabir, M; Faure, D; Heulin, T; Achoawk, W; Bally R. 1995. Oligonucleotide probes based on 16S rRNA sequences for the identification of four Azospirillum species. Can. J. Microbiol 41:1081-1087.

Khan, MS; Zaidi, A; Wani, PA; Ahemad, M; Oves, M. 2009. Functional diversity among plant growth-promoting rhizobacteria: current status. Microbial Strategies for Crop Improvement. Cap6. Berlin Heidelberg, Deushland, Springer-Verlag. p. 105-131.

Khan, MS; Zaidi, A; Ahemad, M; Oves, M; Wani, PA. 2010. Plant growth promotion by phosphate solubilizing fungi - current perspective. Arch Agron Soil Scien 56(1):73-98.

Metha, S; Nautiyal, S. 2001. An efficient method for qualitative screening of phosphate-solubilizing bacteria. Current Microbiology 43:51-55.

Metha, P; Chauhan, A; Mahajan, R; Mahajan, PK; Shirkot, CK. 2010. Strain of Bacillus circulans isolated from apple rhizosphere showing plant growth promoting potential. Current Science 98(4):538-542.

Mikanova, O; Kubat, J; Simon, T; Vorisek, K; Randova, D. 1997. Influence of soluble phosphate on P-solubilizing activity of bacteria. Rostlinna-Vyroba-UZPI 43:421-424.

Muñoz-Rojas, J; Caballero-Mellado, J. 2003. Population dynamics of Gluconacetobacter diazotrophicus in sugarcane cultivars and its effects on plant growth. Microb. Ecol. 46:454-464.

Nautiyal, SC. 1999. An efficient microbiological growth medium for screening phosphate solubilizing microorganism. FEMS Microbiology Letters 170:265275.

NCCLS (Natural Committee for Clinical Laboratory Standards). 1993. Performance standards for antimicrobial disk. Susceptibility test. Approved standard. p. 238.

Patiño, CO. 2010. Solubilización de fosfatos por poblaciones bacterianas aisladas de un suelo del valle del cauca. Estudio de biodiversidad y eficiencia. Tesis Doctorado en Ciencias Agropecuarias Línea de Investigación Manejo de Suelos y Aguas. Universidad Nacional de Colombia, Colombia. 133 p.

Qureshi, MA; Ahmad, ZA; Akhtar, N; Iqbal, A; Mujeeb, F; Shakir, MA. 2012. Role of phosphate solubilizing bacteria (PSB) in enhancing $\mathrm{P}$ availability and promoting cotton growth. The Journal of Animal \& Plant Sciences 22(1):204-210. 
Rajankar, PN; Tambekar, DH; Wate, SR. 2007. Study of phosphate solubilization efficiences of fungi and bacteria isolated from saline belt of Purna river basin. Research Journal of Agriculture and Biological Sciences 3(6):701-703.

Rengel, Z; Marschner P. 2005. Nutrient availability in the rizosphere: exploiting genotypic differences. New Phytologyst 168:305-312.

Rives, N; Hernández, A; Acebo, Y; Heydrich, M. 2006. Caracterización de algunos géneros bacterianos asociados al cultivo del arroz variedad J-104. Revista Cubana del Arroz 2(3):7-13.

Rodríguez, H; Fraga, R. 1999. Phosphate solubilizing bacteria and their role in plant growth promotion. Biotechnology Advances 17:319-339.

Salimpour, S; Khavazi, K; Nadian, H; Besharati, H; Miransari, M. 2010. Enhancing phosphorous availability to canola (Brassica napus L.) using $\mathrm{P}$ solubilizing and sulfur oxidizing bacteria. Aust. J. Crop Sci. 4(5):330-334.

Sharma, K; Dak, G; Agrawal, A; Bhatnagar, M; Sharma, R. 2007. Effect of phosphate solubilizing bacteria on the germination of Cicer arietinum seeds and seedling growth. Journal of Herbal Medicine and Toxicology 1(1):61-63.

United Nations Population Fund. 2011. The state of world population 2011: people and possibilities in a world of 7 billion. New York, USA. $124 \mathrm{p}$.

Vázquez, P; Holguin, G; Puente, ME; López-Cortés, A; Bashan, Y. 2000. Phosphate-solubilizing microorganisms associated with the rhizosphere of mangroves in a semiarid coastal lagoon. Biol Fertil Soils 30:460-468.

Witt, C; Pasuquin, JM; Sulewsk, G. 2009. Predicting agronomic boundaries of future fertilizer needs in AgriStats. Better Crops 93:16-18. 